$\triangle$ GUT MICROBIOTA

\title{
Culturomics: illuminating microbial dark matter
}

'Culturomics' uses multiple highthroughput culture conditions with mass spectroscopy or $16 \mathrm{~S}$ ribosomal RNA sequencing for the identification of previously unculturable bacterial species (often termed 'dark matter'). Now, the culturomics process has been further refined and used to double the number of bacterial species isolated from the human gut.

Metagenomics has revolutionized the study of the human microbiome, but obtaining DNA sequences that cannot be attributed to known bacterial species remains a problem. Pure cultures of microorganisms are also required for extensive microbiology research but many species are resistant to classic culturebased techniques. Culturomics aims to overcome these problems and to fill in gaps in our knowledge of the human microbiome. Lagier and colleagues have now reduced and optimized the culturomics workload and addressed the weaknesses of previous studies. "Our aim in this work was to extend the human gut bacteria repertoire as much as possible," explains author Didier Raoult.

First, the investigators deduced the 18 best culture conditions and implemented new protocols (fresh-sample inoculation and microcolony detection) with a focus on the culture of Proteobacteria and halophilic or microaerophilic bacteria. Using the refined conditions with a range of stool, small bowel and colonic samples from healthy individuals and patients with various diseases from different geographical origins, 1,057 prokaryotic species were isolated, including 197 potentially new species.

By genome sequencing the new species and comparing the data with metagenomic studies from the Human Microbiome Project, the researchers confirmed that culturomics enables the culture of microorganisms that correspond to previously unassigned sequences. "We demonstrated that culturomics is able to fill a part of the [microbial] dark matter," reports Raoult. "These efforts, by increasing knowledge of the [gut microbiota] repertoire, will allow detection of potential probiotics for therapeutic approaches." The investigators also plan to apply culturomics to other human samples beyond the gut.

Iain Dickson

ORIGINAL ARTICLE Lagier, J.-C. et al. Culture of previously uncultured members of the human gut microbiota by culturomics. Nat. Microbiol. http:// dx.doi.org/10.1038/nmicrobiol.2016.203 (2016) 